\title{
MIXED CHORD-INTEGRALS OF STAR BODIES
}

\author{
Lu Fenghong
}

Abstract. The mixed chord-integrals are defined. The Fenchel-Aleksandrov inequality and a general isoperimetric inequality for the mixed chordintegrals are established. Furthermore, the dual general Bieberbach inequality is presented. As an application of the dual form, a BrunnMinkowski type inequality for mixed intersection bodies is given.

\section{Introduction and main results}

In $[6,9]$ Lutwak posed the notion of the mixed width-integrals of convex bodies (compact, convex subsets with non-empty interiors) and obtained a great many properties in common with the dual quermassintegrals (see [7]). It exists closely relations (see $[5,16]$ ) between the mixed width-integrals and mixed projection bodies (see $[10,12]$ ). In [8] Lutwak established a general Bieberbach inequality. Motivated by the ideas of Lutwak, we shall introduce the definitions of the mixed chord-integrals of star bodies and establish a dual general Bieberbach inequality.

The setting for this paper is $n$-dimensional Euclidean space $\mathbb{R}^{n}$. Let $\mathcal{K}^{n}$ denote the set of convex bodies (compact, convex subsets with non-empty interiors) and $\mathcal{K}_{o}^{n}$ denote the subset of $\mathcal{K}^{n}$ that contains the origin in their interiors in $\mathbb{R}^{n}$. Let $S^{n-1}$ denote the unit sphere in $\mathbb{R}^{n}$. The volume of the unit $n$ ball, $U$, will be denoted by $\omega_{n}$. If $K \in \mathcal{K}^{n}$, then the support function of $K$, $h_{K}=h(K, \cdot): \mathbb{R}^{n} \longrightarrow(0, \infty)$, is defined by

$$
h(K, u)=\max \{u \cdot x: x \in K\}, \quad u \in S^{n-1}
$$

where $u \cdot x$ denotes the standard inner product of $u$ and $x$. For $K \in \mathcal{K}^{n}$ and $u \in S^{n-1}, b(K, u)$ is half the width of $K$ in the direction $u$.

Received May 14, 2008; Revised December 15, 2008.

2000 Mathematics Subject Classification. 52A40.

Key words and phrases. mixed intersection bodies, mixed chord-integrals, FenchelAleksandrov inequality, Bieberbach inequality.

Supported by the Sciences Foundation of Shanghai (No.071605123) and by Innovation Program of Shanghai Municipal Education Commission (No.10YZ160). 
Mixed width-integrals, $A\left(K_{1}, \ldots, K_{n}\right)$, of $K_{1}, \ldots, K_{n} \in \mathcal{K}^{n}$ was defined by Lutwak (see [9])

$$
A\left(K_{1}, \ldots, K_{n}\right)=\frac{1}{n} \int_{S^{n-1}} b\left(K_{1}, u\right) \cdots b\left(K_{n}, u\right) d S(u),
$$

where $d S$ is the $(n-1)$-dimensional volume element on $S^{n-1}$. More in general, for a real number $p \neq 0$, the mixed width-integrals of order $p, A_{p}\left(K_{1}, \ldots, K_{n}\right)$, of $K_{1}, \ldots, K_{n} \in \mathcal{K}^{n}$ was also defined by Lutwak (see [9])

$$
A_{p}\left(K_{1}, \ldots, K_{n}\right)=\omega_{n}\left[\frac{1}{n \omega_{n}} \int_{S^{n-1}} b\left(K_{1}, u\right)^{p} \cdots b\left(K_{n}, u\right)^{p} d S(u)\right]^{1 / p},
$$

Furthermore, Lutwak in [9] showed an isoperimetric inequality involving the mixed width-integrals which generalized an inequality obtained by Chakerian $($ see $[1,2])$.

Theorem 1*. If $K_{1}, \ldots, K_{n} \in \mathcal{K}^{n}$, then

$$
V\left(K_{1}\right) \cdots V\left(K_{n}\right) \leq A^{n}\left(K_{1}, \ldots, K_{n}\right),
$$

with equality if and only if $K_{1}, K_{2}, \ldots, K_{n}$ are $n$-balls.

A more general version of inequality (1.4) is obtained by introducing indexed mixed width-integrals.

Theorem 2*. If $K_{1}, \ldots, K_{n} \in \mathcal{K}^{n}, p \neq 0$ and $-1 \leq p \leq \infty$, then

$$
V\left(K_{1}\right) \cdots V\left(K_{n}\right) \leq A_{p}^{n}\left(K_{1}, \ldots, K_{n}\right),
$$

with equality if and only if $K_{1}, K_{2}, \ldots, K_{n}$ are $n$-balls.

Let $\mathcal{S}_{o}^{n}$ denote the set of star bodies in $\mathbb{R}^{n}$ containing the origin in their interiors. In this paper, we give the definitions of the mixed chord-integrals, $B\left(L_{1}, \ldots, L_{n}\right)$ and for a real number $p \neq 0$, the mixed chord-integrals of order $p, B_{p}\left(L_{1}, \ldots, L_{n}\right)$, of $L_{1}, \ldots, L_{n} \in \mathcal{S}_{o}^{n}$ and the $p$-chord, $\tilde{d}_{p}(L)$, of $L \in \mathcal{S}_{o}^{n}$, respectively. We mainly obtain the following results.

The analog of Theorem $1^{*}$ (i.e., the general isoperimetric inequality involving the mixed width-integrals) for the mixed chord-integrals is obtained:

Theorem 1. If $L_{1}, \ldots, L_{n} \in \mathcal{S}_{o}^{n}$, then

$$
B^{n}\left(L_{1}, \ldots, L_{n}\right) \leq V\left(L_{1}\right) \cdots V\left(L_{n}\right),
$$

with equality if and only if $L_{1}, \ldots, L_{n}$ are dilates and centered.

A more general version of inequality (1.6) is obtained:

Theorem 2. If $L_{1}, \ldots, L_{n} \in \mathcal{S}_{o}^{n}$ and $-\infty \leq p \leq 1$, then

$$
B_{p}^{n}\left(L_{1}, \ldots, L_{n}\right) \leq V\left(L_{1}\right) \cdots V\left(L_{n}\right),
$$

with equality if and only if $L_{1}, \ldots, L_{n}$ are $n$-balls. 
Theorem 3. If $L_{1}, \ldots, L_{n} \in \mathcal{S}_{o}^{n}$ and $1<m \leq n$, then

$$
B^{m}\left(L_{1}, \ldots, L_{n}\right) \leq \prod_{i=0}^{m-1} B\left(L_{1}, \ldots, L_{n-m}, L_{n-i}, \ldots, L_{n-i}\right),
$$

with equality if and only if $L_{n-m+1}, L_{n-m+2}, \ldots, L_{n}$ are all of similar chord.

A more general version of inequality (1.8) is obtained:

Theorem 4. If $L_{1}, \ldots, L_{n} \in \mathcal{S}_{o}^{n}$ and $1<m \leq n$, then for $p>0$

$$
B_{p}^{m}\left(L_{1}, \ldots, L_{n}\right) \leq \prod_{i=0}^{m-1} B_{p}\left(L_{1}, \ldots, L_{n-m}, L_{n-i}, \ldots, L_{n-i}\right),
$$

with equality if and only if $L_{n-m+1}, L_{n-m+2}, \ldots, L_{n}$ are all of similar chord. For $p<0$, inequality (1.9) is reversed.

Theorem 3 and Theorem 4 are just analogs of the Fenchel-Aleksandrov inequality for the dual mixed volumes (see [7]).

Moreover, we obtain the dual general Bieberbach inequality as follows.

Theorem 5. If $L \in \mathcal{S}_{o}^{n}$ and $-\infty \leq p<n$, then

$$
V(L) \geq \omega_{n} \tilde{d}_{p}(L)^{n},
$$

with equality if and only if $L$ is an $n$-ball.

Theorem 5 is just a dual form of the following general Bieberbach inequality which was shown by Lutwak [8].

Theorem $5^{*}$. If $K \in \mathcal{K}^{n}$ and $-n<p \leq \infty$, then

$$
V(K) \leq \omega_{n} \bar{b}_{p}(K)^{n},
$$

with equality if and only if $K$ is an $n$-ball.

As an application of Theorem 5, we establish a Brunn-Minkowski type inequality for mixed intersection bodies which defined in $[11,15]$.

Theorem 6. If $L_{1}, L_{2} \in \mathcal{S}_{o}^{n}$ and $i \leq n-1$, then

$$
\tilde{W}_{i}\left(I\left(L_{1} \breve{+} L_{2}\right)\right)^{1 /(n-i)} \leq \tilde{W}_{i}\left(I L_{1}\right)^{1 /(n-i)}+\tilde{W}_{i}\left(I L_{2}\right)^{1 /(n-i)},
$$

with equality if and only if $L_{1}$ and $L_{2}$ are dilates. If $n-1<i<n$ or $i>n$, then this inequality is reversed.

In particular, for $i=0$, we obtain:

Corollary 1. If $L_{1}, L_{2} \in \mathcal{S}_{O}^{n}$, then

$$
V\left(I\left(L_{1} \breve{+} L_{2}\right)\right)^{1 / n} \leq V\left(I L_{1}\right)^{1 / n}+V\left(I L_{2}\right)^{1 / n},
$$

with equality if and only if $L_{1}$ and $L_{2}$ are dilates. 


\section{Mixed chord-integrals}

For a compact subset $L$ of $\mathbb{R}^{n}$, which is star-shaped with respect to the origin, we shall use $\rho(L, \cdot)$ to denote its radial function; i.e., for $u \in S^{n-1}$,

$$
\rho(L, u)=\max \{\lambda>0: \lambda u \in L\} .
$$

If $\rho(L, \cdot)$ is continuous and positive, $L$ will be called a star body. Two star bodies $K$ and $L$ are said to be dilates if $\rho_{K}(u) / \rho_{L}(u)$ is independent of $u \in$ $S^{n-1}$.

If $K \in \mathcal{K}_{o}^{n}$, the polar body of $K, K^{*}$, is defined by

$$
K^{*}=\left\{x \in \mathbb{R}^{n}: x \cdot y \leq 1, x \in K\right\} .
$$

Obviously, we have $\left(K^{*}\right)^{*}=K$. From the definition (2.2), we also know that: If $K \in \mathcal{K}_{o}^{n}$, then the support and radial function of $K^{*}$, the polar body of $K$, are respectively defined by

$$
h_{K^{*}}=\frac{1}{\rho_{K}} \text { and } \rho_{K^{*}}=\frac{1}{h_{K}} .
$$

The polar coordinate formula for volume of body $L$ in $\mathbb{R}^{n}$ is

$$
V(L)=\frac{1}{n} \int_{S^{n-1}} \rho(L, u)^{n} d S(u) .
$$

If $L \in \mathcal{S}_{o}^{n}$ and $u \in S^{n-1}$, we let

$$
d(L, u)=\frac{1}{2} \rho(L, u)+\frac{1}{2} \rho(L,-u),
$$

i.e., $d(L, u)$ denotes half the chord of $L$ in the direction $u$. Star bodies $L_{1}, \ldots, L_{n}$ are said to have similar chord if there exist constants $\lambda_{1}, \ldots, \lambda_{n}>0$ such that $\lambda_{1} d\left(L_{1}, u\right)=\cdots=\lambda_{n} d\left(L_{n}, u\right)$ for all $u \in S^{n-1}$; they are said to have constant chord jointly if the product $d\left(L_{1}, u\right) \cdots d\left(L_{n}, u\right)$ is constant for all $u \in S^{n-1}$. For reference see Gardner ([3]) and schneider ([14]).

Following Lutwak, we define the mixed chord integral of star bodies: For $L_{1}, \ldots, L_{n} \in \mathcal{S}_{o}^{n}$, the mixed chord-integral, $B\left(L_{1}, \ldots, L_{n}\right)$, of $L_{1}, \ldots, L_{n}$ is defined by

$$
B\left(L_{1}, \ldots, L_{n}\right)=\frac{1}{n} \int_{S^{n-1}} d\left(L_{1}, u\right) \cdots d\left(L_{n}, u\right) d S(u) .
$$

By this definition, $B$ is a map

$$
B: \underbrace{\mathcal{S}_{o}^{n} \times \cdots \times \mathcal{S}_{o}^{n}}_{n} \longrightarrow \mathbb{R}
$$

We list some of its elementary properties.

(i) (Continuity) The mixed chord-integral $B\left(L_{1}, \ldots, L_{n}\right)$ is a continuous function of $L_{1}, \ldots, L_{n} \in \mathcal{S}_{o}^{n}$.

(ii) (Positivity) For $L_{1}, \ldots, L_{n} \in \mathcal{S}_{o}^{n}, B\left(L_{1}, \ldots, L_{n}\right)>0$.

(iii) (Positively homogeneous) If $L_{1}, \ldots, L_{n} \in \mathcal{S}_{o}^{n}$ and $\lambda_{1}, \ldots, \lambda_{n}>0$, then

$$
B\left(\lambda_{1} L_{1}, \ldots, \lambda_{n} L_{n}\right)=\lambda_{1} \cdots \lambda_{n} B\left(L_{1}, \ldots, L_{n}\right) .
$$


(iv) (Monotonicity for set inclusion) If $K_{i}, L_{i} \in \mathcal{S}_{o}^{n}, K_{i} \subset L_{i}$ and $1 \leq i \leq n$, then

$$
B\left(K_{1}, \ldots, K_{n}\right) \leq B\left(L_{1}, \ldots, L_{n}\right),
$$

with equality if and only if $K_{i}=L_{i}$ for $1 \leq i \leq n$.

(v) (Change under linear transformations) If $L_{1}, \ldots, L_{n} \in \mathcal{S}_{o}^{n}$ and $\phi \in$ $G L(n)$, then

$$
B\left(\phi L_{1}, \ldots, \phi L_{n}\right)=|\operatorname{det} \phi| B\left(L_{1}, \ldots, L_{n}\right) .
$$

Just as the width-integrals $B_{i}(K)$ (see [6]) of $K \in \mathcal{K}^{n}$, are defined to be the special mixed width-integrals

$$
A(\underbrace{K, \ldots, K}_{n-i}, \underbrace{U, \ldots, U}_{i})
$$

the chord-integrals $D_{i}(L)$ of $L \in \mathcal{S}_{o}^{n}$, can be defined as the special mixed chordintegral

$$
B(\underbrace{L, \ldots, L}_{n-i}, \underbrace{U, \ldots, U}_{i})
$$

Now we generalize the notion of the mixed chord-integral of star bodies: For $L_{1}, \ldots, L_{n} \in \mathcal{S}_{o}^{n}$ and a real number $p \neq 0$, the mixed chord-integral of order $p$, $B_{p}\left(L_{1}, \ldots, L_{n}\right)$, of $L_{1}, \ldots, L_{n}$ is defined by

$$
B_{p}\left(L_{1}, \ldots, L_{n}\right)=\omega_{n}\left[\frac{1}{n \omega_{n}} \int_{S^{n-1}} d\left(L_{1}, u\right)^{p} \cdots d\left(L_{n}, u\right)^{p} d S(u)\right]^{1 / p} .
$$

Specially $p=1$, then definition (2.7) is just definition (2.6). For $p$ equal to $-\infty, 0$ or $\infty$ we respectively define the mixed chord-integral of order $p$ by

$$
B_{p}\left(L_{1}, \ldots, L_{n}\right)=\lim _{s \rightarrow p} B_{s}\left(L_{1}, \ldots, L_{n}\right) .
$$

As a direct consequence of Jensen's inequality [4] we have:

Proposition 1. If $L_{1}, \ldots, L_{n} \in \mathcal{S}_{o}^{n}$ and $-\infty \leq p<q \leq \infty$, then

$$
B_{p}\left(L_{1}, \ldots, L_{n}\right) \leq B_{q}\left(L_{1}, \ldots, L_{n}\right),
$$

with equality if and only if $L_{1}, \ldots, L_{n}$ have constant chord jointly.

The well-known Blaschke-Santaló inequality (see [13]) can be stated: For $K \in \mathcal{K}_{o}^{n}$, then

$$
V(K) V\left(K^{*}\right) \leq \omega_{n}^{2},
$$

with equality if and only if $K$ is an $n$-dimensional ellipsoid.

By combining the well-known Blaschke-Santaló inequality with Theorem 2, we obtain the Blaschke-Santaló type inequality for the mixed chord-integral of order $p$ (the mixed chord-integral when $p=1$ ).

Corollary 2. If $K_{1}, \ldots, K_{n} \in \mathcal{K}_{o}^{n}$, then

$$
B_{p}\left(K_{1}, \ldots, K_{n}\right) B_{p}\left(K_{1}^{*}, \ldots, K_{n}^{*}\right) \leq \omega_{n}^{2},
$$

with equality if and only if $K_{1}, \ldots, K_{n}$ are $n$-balls. 
In particular, if $L_{1}=\cdots=L_{n-i}=L$ and $L_{n-i+1}=\cdots=L_{n}=U$, then Theorem 1 becomes:

Corollary 3. If $L \in \mathcal{S}_{o}^{n}$ and $0 \leq i \leq n$, then

$$
D_{i}^{n}(L) \leq \omega_{n}^{i} V(L)^{n-i},
$$

with equality if and only if $L$ is an $n$-ball.

Combing Theorems $1^{*}$ and $2^{*}$ with Theorems 1 and 2, we obtain the following relations between $B\left(L_{1}, \ldots, L_{n}\right), B_{p}\left(L_{1}, \ldots, L_{n}\right)$ and $A\left(L_{1}, \ldots, L_{n}\right)$, $A_{p}\left(L_{1}, \ldots, L_{n}\right)$, respectively.

Corollary 4. If $K_{1}, \ldots, K_{n} \in \mathcal{K}^{n}$, then

$$
B\left(K_{1}, \ldots, K_{n}\right) \leq A\left(K_{1}, \ldots, K_{n}\right),
$$

with equality if and only if $K_{1}, K_{2}, \ldots, K_{n}$ are $n$-balls.

Corollary 5. If $K_{1}, \ldots, K_{n} \in \mathcal{K}^{n}$ and $-1 \leq p \leq 1$, then

$$
B_{p}\left(K_{1}, \ldots, K_{n}\right) \leq A_{p}\left(K_{1}, \ldots, K_{n}\right),
$$

with equality if and only if $K_{1}, K_{2}, \ldots, K_{n}$ are $n$-balls.

Proof of Theorem 1. For $L_{1}, \ldots, L_{n} \in \mathcal{S}_{o}^{n}$. From definition (2.6), Hölder integral inequality [4], definition (2.5), Minkowski integral inequality [4] and formula (2.4), we have

$$
\begin{aligned}
& B\left(L_{1}, \ldots, L_{n}\right) \\
= & \frac{1}{n} \int_{S^{n-1}} d\left(L_{1}, u\right) \cdots d\left(L_{n}, u\right) d S(u) \\
\leq & n^{-1 / n}\left\|d\left(L_{1}, u\right)\right\|_{n} \cdots n^{-1 / n}\left\|d\left(L_{n}, u\right)\right\|_{n} \\
= & n^{-1 / n}\left\|\frac{1}{2} \rho\left(L_{1}, u\right)+\frac{1}{2} \rho\left(L_{1},-u\right)\right\|_{n} \cdots n^{-1 / n}\left\|\frac{1}{2} \rho\left(L_{n}, u\right)+\frac{1}{2} \rho\left(L_{n},-u\right)\right\|_{n} \\
\leq & n^{-1 / n}\left\|\rho\left(L_{1}, u\right)\right\|_{n} \cdots n^{-1 / n}\left\|\rho\left(L_{n}, u\right)\right\|_{n} \\
= & V^{\frac{1}{n}}\left(L_{1}\right) \cdots V^{\frac{1}{n}}\left(L_{n}\right) .
\end{aligned}
$$

In view of the equality conditions of Hölder integral inequality and Minkowski integral inequality, equality of inequality (1.6) holds if and only if $L_{1}, \ldots, L_{n}$ are dilates and centered. Thus we obtain the conclusion.

Proof of Theorem 2. For $L_{1}, \ldots, L_{n} \in \mathcal{S}_{o}^{n}$ and $-\infty \leq p \leq 1$. By combining Theorem 1 with Proposition 1 we obtain

$$
\begin{aligned}
B_{p}^{n}\left(L_{1}, \ldots, L_{n}\right) & \leq B_{1}^{n}\left(L_{1}, \ldots, L_{n}\right) \\
& \leq V\left(L_{1}\right) \cdots V\left(L_{n}\right) .
\end{aligned}
$$

In view of the equality conditions of Theorem 1 and Proposition 1, equality holds if and only if $L_{1}, \ldots, L_{n}$ are $n$-balls. Thus we obtain the conclusion. 
In order to prove Theorem 3 and Theorem 4 in the introduction, we require the following simple extension of Hölder's inequality.

Lemma 1 (see [7]). If $f_{0}, f_{1}, \ldots, f_{m}$ are (strictly) positive continuous functions defined on $S^{n-1}$ and $\lambda_{1}, \ldots, \lambda_{m}$ are positive constants the sum of whose reciprocals is unity, then

$$
\int_{S^{n-1}} f_{0}(u) f_{1}(u) \cdots f_{m}(u) d S(u) \leq \prod_{i=1}^{m}\left[\int_{S^{n-1}} f_{0}(u) f_{i}^{\lambda_{i}}(u) d S(u)\right]^{1 / \lambda_{i}},
$$

with equality if and only if there exist positive constants $\alpha_{1}, \ldots, \alpha_{m}$ such that $\alpha_{1} f_{1}^{\lambda_{1}}(u)=\cdots=\alpha_{m} f_{m}^{\lambda_{m}}(u)$ for all $u \in S^{n-1}$.

Proof of Theorem 3. For $L_{1}, \ldots, L_{n} \in \mathcal{S}_{o}^{n}$. Let

$$
\begin{aligned}
\lambda_{i} & =m(1 \leq i \leq m), \\
f_{0} & =d\left(L_{1}, u\right) \cdots d\left(L_{n-m}, u\right)\left(f_{0}=1 \text { if } m=n\right), \\
f_{i} & =d\left(L_{n-i+1}, u\right)(1 \leq i \leq m) .
\end{aligned}
$$

Using Lemma 1, we have

$$
\begin{aligned}
& \int_{S^{n-1}} d\left(L_{1}, u\right) \cdots d\left(L_{n}, u\right) d S(u) \\
\leq & \prod_{i=1}^{m}\left[\int_{S^{n-1}} d\left(L_{1}, u\right) \cdots d\left(L_{n-m}, u\right) d\left(L_{n-i+1}, u\right)^{m} d S(u)\right]^{1 / \lambda_{i}},
\end{aligned}
$$

with equality if and only if $L_{n-m+1}, L_{n-m+2}, \ldots, L_{n}$ are all of similar chord. Thus we obtain the conclusion.

Proof of Theorem 4. For $L_{1}, \ldots, L_{n} \in \mathcal{S}_{o}^{n}$. Let

$$
\begin{aligned}
\lambda_{i} & =m(1 \leq i \leq m), \\
f_{0} & =d\left(L_{1}, u\right)^{p} \cdots d\left(L_{n-m}, u\right)^{p}\left(f_{0}=1 \text { if } m=n\right), \\
f_{i} & =d\left(L_{n-i+1}, u\right)^{p}(1 \leq i \leq m) .
\end{aligned}
$$

Using Lemma 1, we have

$$
\begin{aligned}
& \int_{S^{n-1}} d\left(L_{1}, u\right)^{p} \cdots d\left(L_{n}, u\right)^{p} d S(u) \\
\leq & \prod_{i=1}^{m}\left[\int_{S^{n-1}} d\left(L_{1}, u\right)^{p} \cdots d\left(L_{n-m}, u\right)^{p} d\left(L_{n-i+1}, u\right)^{p m} d S(u)\right]^{1 / \lambda_{i}},
\end{aligned}
$$

with equality if and only if $L_{n-m+1}, L_{n-m+2}, \ldots, L_{n}$ are all of similar chord.

For $p>0$, we get

$$
\begin{aligned}
& \omega_{n}\left[\frac{1}{n \omega_{n}} \int_{S^{n-1}} d\left(L_{1}, u\right)^{p} \cdots d\left(L_{n}, u\right)^{p} d S(u)\right]^{1 / p} \\
\leq & \omega_{n} \prod_{i=1}^{m}\left[\frac{1}{n \omega_{n}} \int_{S^{n-1}} d\left(L_{1}, u\right)^{p} \cdots d\left(L_{n-m}, u\right)^{p} d\left(L_{n-i+1}, u\right)^{p} d S(u)\right]^{1 / p \lambda_{i}},
\end{aligned}
$$


with equality if and only if $L_{n-m+1}, L_{n-m+2}, \ldots, L_{n}$ are all of similar chord. For $p<0$, inequality above is reversed.

Thus we obtain the conclusion.

\section{Dual general Bieberbach inequality}

In [8] Lutwak established a general Bieberbach inequality which has the Bieberbach, Urysohn and harmonic Urysohn inequalities as special cases. Following Lutwak, we give a dual general Bieberbach inequality.

If $L \in \mathcal{S}_{o}^{n}$ and $i \in \mathbb{R}$, then the $i$-th dual quermassintegrals is defined by Lutwak (see [7])

$$
\tilde{W}_{i}(L)=\frac{1}{n} \int_{S^{n-1}} \rho(L, u)^{n-i} d S(u) .
$$

Specifically, $\tilde{W}_{0}(K)=V(K)$, and $\tilde{W}_{n}(K)=\omega_{n}$.

For $L \in \mathcal{S}_{o}^{n}$, the intersection body of $L, I L$ is the centrally symmetric body whose radial function on $S^{n-1}$ is given by (see [11]),

$$
\rho(I L, u)=\frac{1}{n-1} \int_{S^{n-1} \cap u^{\perp}} \rho(L, v)^{n-1} d \lambda_{n-2}(v),
$$

where $\lambda_{n-2}$ denotes $(n-2)$-dimensional Lebesgue measure. For $u \in S^{n-1}$, $L \cap u^{\perp}$ denotes the intersection of $L$ with the subspace $u^{\perp}$ that passes through the origin and is orthogonal to $u$.

For $L_{1}, L_{2} \in \mathcal{S}_{o}^{n}$, the radial addition $L_{1} \tilde{+} L_{2}$ is defined as the star body whose radial function is given by (see [11]),

$$
\rho\left(L_{1} \tilde{+} L_{2}, \cdot\right)=\rho\left(L_{1}, \cdot\right)+\rho\left(L_{2}, \cdot\right) .
$$

The radial Blaschke linear addition, $\lambda \cdot K \breve{+} \mu \cdot L$, is defined by Lutwak (see [11]), whose radial function satisfies for $u \in S^{n-1}$ (see [11])

$$
\rho(\lambda \cdot K \breve{+} \mu \cdot L, u)^{n-1}=\lambda \rho(K, u)^{n-1}+\mu \rho(L, u)^{n-1} .
$$
then

$$
\begin{gathered}
d\left(L_{1} \tilde{+} L_{2}, \cdot\right)=d\left(L_{1}, \cdot\right)+d\left(L_{2}, \cdot\right), \\
I(\lambda \cdot K \breve{+} \mu \cdot L)=\lambda I K \tilde{+} \mu I L .
\end{gathered}
$$

For $K \in \mathcal{K}^{n}$ and a real number $p \neq 0$, the $p$-width, $\bar{b}_{p}(K)$ of $K$ was defined by Lutwak in $([8])$

$$
\bar{b}_{p}(K)=\left[\frac{1}{n \omega_{n}} \int_{S^{n-1}} b^{p}(K, u) d S(u)\right]^{1 / p},
$$

where the definition (3.6) differs slightly from that of Lutwak (see [8]) in that we multiply a constant factor. For $p$ equal to $-\infty, 0$ or $\infty$ the $p$-width of $K$ was defined by

$$
\bar{b}_{p}(K)=\lim _{s \rightarrow p} \bar{b}_{s}(K) .
$$


In order to establish the dual general Bieberbach inequality, we shall also introduce the dual concept of the $p$-width of convex body: For $L \in \mathcal{S}_{o}^{n}$ and a real number $p \neq 0$, the $p$-chord, $\tilde{d}_{p}(L)$ of $L$ was defined by

$$
\tilde{d}_{p}(L)=\left[\frac{1}{n \omega_{n}} \int_{S^{n-1}} d^{p}(L, u) d S(u)\right]^{1 / p} .
$$

For $p$ equal to $-\infty, 0$ or $\infty$ the $p$-chord of $L$ was defined by

$$
\tilde{d}_{p}(L)=\lim _{s \rightarrow p} \tilde{d}_{s}(L) .
$$

For a fixed $p$, the $p$-chord is a map

$$
\tilde{d}_{p}: \mathcal{S}_{o}^{n} \longrightarrow \mathbb{R}
$$

Similar to the mixed chord-integral, it is positive, continuous, bounded, homogeneous of degree one and monotone under set inclusion.

From Theorem 5 and Theorem $5^{*}$ we can obtain the relation between the dual general Bieberbach inequality and the general Bieberbach inequality as follows.

Corollary 6. If $K \in \mathcal{K}^{n}$ and $-n<p<n$, then

$$
\tilde{d}_{p}(K) \leq \bar{b}_{p}(K),
$$

with equality if and only if $K$ is an $n$-ball.

We shall prove the following relation between the $p$-chord of convex body and the $p$-chord of polar for convex body.

Theorem 7. If $K \in \mathcal{K}_{o}^{n}$ and $-\infty \leq p<n$, then

$$
\tilde{d}_{p}(K) \tilde{d}_{p}\left(K^{*}\right) \leq 1,
$$

with equality if and only if $K$ is an $n$-ball.

Theorem 7 is just the dual of the following relation between the $p$-width of convex body and the $p$-width of polar for convex body which was shown by Lutwak (see [8]).

Theorem $7^{*}$. If $K \in \mathcal{K}^{n}$ and $-n<p \leq \infty$, then

$$
\bar{b}_{p}(K) \bar{b}_{p}\left(K^{*}\right) \geq 1 \text {, }
$$

with equality if and only if $K$ is an $n$-ball.

Furthermore, we establish the following Brunn-Minkowski inequality for the $p$-chord of star bodies.

Theorem 8. For $L_{1}, L_{2} \in \mathcal{S}_{o}^{n}, p \geq 1$ and $\alpha \in[0,1]$, then

$$
\begin{aligned}
\tilde{d}_{p}\left(L_{1} \tilde{+} L_{2}\right) & \leq \tilde{d}_{p}\left(\alpha L_{1} \tilde{+}(1-\alpha) L_{2}\right)+\tilde{d}_{p}\left((1-\alpha) L_{1} \tilde{+} \alpha L_{2}\right) \\
& \leq \tilde{d}_{p}\left(L_{1}\right)+\tilde{d}_{p}\left(L_{2}\right)
\end{aligned}
$$

in each inequalities, with equality if and only if $L_{1}$ and $L_{2}$ have similar chord. If $p<1$ and $p \neq 0$, then inequality (3.8) is reversed. 
Proof of Theorem 5. For $L \in \mathcal{S}_{o}^{n}$, taking $p=n$, from definition (3.7), definition (2.5), Minkowski integral inequality (see [4]) and formula (2.4), we get

$$
\begin{aligned}
\tilde{d}_{n}(L) & =\left[\frac{1}{n \omega_{n}} \int_{S^{n-1}} d^{n}(L, u) d S(u)\right]^{1 / n} \\
& =\left[\frac{1}{n \omega_{n}} \int_{S^{n-1}}\left[\frac{1}{2} \rho(L, u)+\frac{1}{2} \rho(L,-u)\right]^{n} d S(u)\right]^{1 / n} \\
& \leq \omega_{n}^{-1 / n}\left[\frac{1}{n} \int_{S^{n-1}} \rho^{n}(L, u) d S(u)\right]^{1 / n} \\
& =\omega_{n}^{-1 / n} V(L)^{1 / n}
\end{aligned}
$$

with equality if and only if $L$ is centered. From Jensen's inequality (see [4]), it follows that for $-\infty \leq p<n$

$$
\tilde{d}_{n}(L) \geq \tilde{d}_{p}(L)
$$

with equality if and only if $L$ is of constant chord.

Combing these inequalities above, we have

$$
V(L) \geq \omega_{n} \tilde{d}_{p}(L)^{n},
$$

with equality if and only if $L$ is an $n$-ball.

Proof of Theorem \%. For $K \in \mathcal{K}_{o}^{n}$, combing inequality (3.9) with BlaschkeSantaló inequality, we obtain

$$
\omega_{n} \tilde{d}_{n}(K)^{-n} \geq V\left(K^{*}\right),
$$

with equality if and only if $L$ is an $n$-ball. By combing inequality (3.10) and inequality (3.11), we have for $-\infty \leq p<n$,

$$
\omega_{n} \tilde{d}_{p}(K)^{-n} \geq V\left(K^{*}\right),
$$

with equality if and only if $L$ is an $n$-ball.

According to the inequality (3.11), inequality (3.12) and the dual general Bieberbach inequality, we obtain the desired result.

Proof of Theorem 8. For $L_{1}, L_{2} \in \mathcal{S}_{o}^{n}, p \geq 1$ and $\alpha \in[0,1]$. From definition (3.7) and formula (3.4), Minkowski integral inequality, definition (3.7) and (3.4) again, Minkowski integral inequality again, definition (3.7) again, it follows that

$$
\begin{aligned}
\tilde{d}_{p}\left(L_{1} \tilde{+} L_{2}\right) & =\left(n \omega_{n}\right)^{-1 / p}\left\|d\left(L_{1} \tilde{+} L_{2}, u\right)\right\|_{p} \\
& =\left(n \omega_{n}\right)^{-1 / p}\left\|d\left(L_{1}, u\right)+d\left(L_{2}, u\right)\right\|_{p}
\end{aligned}
$$




$$
\begin{aligned}
= & \left(n \omega_{n}\right)^{-1 / p} \|\left[\alpha d\left(L_{1}, u\right)+(1-\alpha) d\left(L_{2}, u\right)\right] \\
& +\left[(1-\alpha) d\left(L_{1}, u\right)+\alpha d\left(L_{2}, u\right)\right] \|_{p} \\
\leq & \left(n \omega_{n}\right)^{-1 / p}\left\|\alpha d\left(L_{1}, u\right)+(1-\alpha) d\left(L_{2}, u\right)\right\|_{p} \\
& \left.+\left(n \omega_{n}\right)^{-1 / p} \|(1-\alpha) d\left(L_{1}, u\right)+\alpha d\left(L_{2}, u\right)\right] \|_{p} \\
= & \tilde{d}_{p}\left(\alpha L_{1} \tilde{+}(1-\alpha) L_{2}\right)+\tilde{d}_{p}\left((1-\alpha) L_{1} \tilde{+} \alpha L_{2}\right) \\
\leq & \left(n \omega_{n}\right)^{-1 / p}\left\|\alpha d\left(L_{1}, u\right)\right\|_{p}+\left(n \omega_{n}\right)^{-1 / p}\left\|(1-\alpha) d\left(L_{2}, u\right)\right\|_{p} \\
& +\left(n \omega_{n}\right)^{-1 / p}\left\|(1-\alpha) d\left(L_{1}, u\right)\right\|_{p}+\left(n \omega_{n}\right)^{-1 / p}\left\|\alpha d\left(L_{2}, u\right)\right\|_{p} \\
= & \tilde{d}_{p}\left(L_{1}\right)+\tilde{d}_{p}\left(L_{2}\right),
\end{aligned}
$$

in each inequalities, with equality if and only if $L_{1}$ and $L_{2}$ have similar chord. In view of the inverse Minkowski integral inequality, similar above the proof, the cases of $p<1$ and $p \neq 0$ can also be proved. Here we omit the details, i.e., if $p<1$ and $p \neq 0$, then this inequality is reversed.

Proof of Theorem 6. For $L_{1}, L_{2} \in \mathcal{S}_{o}^{n}$ and $i \leq n-1$. Since $I\left(L_{1} \breve{+} L_{2}\right), I L_{1}$ and $I L_{2}$ are centered, then

$$
\begin{gathered}
d\left(I\left(L_{1} \breve{+} L_{2}\right), u\right)=\rho\left(I\left(L_{1} \breve{+} L_{2}\right), u\right), \\
d\left(I L_{1}, u\right)=\rho\left(I L_{1}, u\right), d\left(I L_{2}, u\right)=\rho\left(I L_{2}, u\right) .
\end{gathered}
$$

Combing Theorem 8 with definition (3.1) and equalities above, we have

$$
\tilde{W}_{i}\left(I\left(L_{1} \breve{+} L_{2}\right)\right)^{1 /(n-i)} \leq \tilde{W}_{i}\left(I L_{1}\right)^{1 /(n-i)}+\tilde{W}_{i}\left(I L_{2}\right)^{1 /(n-i)},
$$

with equality if and only if $L_{1}$ and $L_{2}$ are dilates. If $n-1<i<n$ or $i>n$, then this inequality is reversed.

Acknowledgements. The author wish to thank the referee(s) for their many excellent suggestions for improving the original manuscript.

\section{References}

[1] G. D. Chakerian, Isoperimetric inequalities for the mean width of a convex body, Geometriae Dedicata 1 (1973), no. 3, 356-362.

[2] - The mean volume of boxes and cylinders circumscribed about a convex body, Israel J. Math. 12 (1972), 249-256.

[3] R. J. Gardner, Geometric Tomography, Cambridge Univ. Press, Cambridge, 1995.

[4] H. Hardy, J. E. Littlewood, and G. Pólya, Inequalities, Cambridge Univ. Press, London, 1934.

[5] G. Leng, C. Zhao, B. He, and X. Li, Inequalities for polars of mixed projection bodies, Sci. China Ser. A 47 (2004), no. 2, 175-186.

[6] E. Lutwak, Width-integrals of convex bodies, Proc. Amer. Math. Soc. 53 (1975), no. 2, 435-439.

[7] _ Dual mixed volumes, Pacific J. Math. 58 (1975), no. 2, 531-538.

[8] __ A general Bieberbach inequality, Math. Proc. Cambridge Philos. Soc. 78 (1975), no. 3, 493-495.

[9] _ Mixed width-integrals of convex bodies, Israel J. Math. 28 (1977), no. 3, 249253. 
[10] — Mixed projection inequalities, Trans. Amer. Math. Soc. 287 (1985), no. 1, 91105.

[11] , Intersection bodies and dual mixed volumes, Adv. in Math. 71 (1988), no. 2, $232-261$

[12] _ Inequalities for mixed projection bodies, Trans. Amer. Math. Soc. 339 (1993), no. 2, 901-916.

[13] L. Santaló, An affine invariant for convex bodies of n-dimensional space, Portugaliae Math. 8 (1949), 155-161.

[14] R. Schneider, Convex Body: The Brunn-Minkowski Theory, Cambridge Univ. Press, Cambridge, 1993.

[15] G. Zhang, Centered bodies and dual mixed volumes, Trans. Amer. Math. Soc. 345 (1994), no. $2,777-801$

[16] C. J. Zhao and G. S. Leng, On polars of mixed projection bodies, J. Math. Anal. Appl. 316 (2006), no. 2, 664-678.

Department of Mathematics

Shanghai University of Electric Power

Shanghai, 200090, P. R. China

E-mail address: lufengred@163.com 